\title{
Evolution of Principles of Transmetatarsal Amputations: How We Do It Today
}

\author{
Deepak Kumar ${ }^{1}$, Aman Hooda $^{2}$, Tarkik Thami ${ }^{3}$
}

\begin{abstract}
Transmetatarsal amputation (TMA) aims to preserve the length of the foot as much as possible in patients with traumatic mangled foot injuries or diabetic foot. In this article, we give an overview of the evolution of technique of TMA, management of complications, its postoperative care, with utmost emphasis on preoperative and intraoperative steps to achieve best results. We have formulated a treatment algorithm as per our institutional practice for systematic application of principles for the management of TMA.

Keywords: Diabetic foot, Limb salvage, Surgery, Transmetatarsal amputation.

Journal of Foot and Ankle Surgery (Asia Pacific) (2020): 10.5005/jp-journals-10040-1129
\end{abstract}

\section{BACKGROUND}

Transmetatarsal amputation (TMA) is defined as amputation of the forefoot through metatarsals, with an aim of preserving as much length of the foot as possible, in order to maximally optimize limb function. ${ }^{1}$ Transmetatarsal amputation was first described by Bernard and Heute ${ }^{2}$ for trench foot in 1855. It gained worldwide recognition in 1949 when McKittrick et al. ${ }^{3}$ used it successfully in conjunction with antibiotic therapy for the management of diabetic foot patients, which holds true even after 70 years. In the 1990s, approximately 10,000 TMAs were performed in the United States, compared with 32,000 above-knee amputations and 22,000 belowknee amputations. ${ }^{4}$ The TMA may have advantages over hindfoot and below-knee amputations, especially in cases of infection limited to the forefoot, local gangrene, and nonhealing ulcers in distal part of the foot. The main advantage is preservation of a weightbearing platform which allows early mobilization and has a better cosmetic appearance. ${ }^{5}$ With evolution of surgical techniques and technology, TMA underwent some variations; efforts were made to minimize potential disability after amputation, which could be in the form of stump coverage, equinus contracture, and issues with footwear. The principle of more proximal the amputation greater the disability, however, remains unchanged to date. Modifications of surgical technique in TMA have evolved in recent years with guillotine metatarsal amputation ${ }^{6}$ or modified $\mathrm{TMA}^{7}$ as available options, but a combination of principles of McKittrick et $\mathrm{al}^{3}$ and improved soft tissue reconstruction is what makes this amputation a viable option even today.

\section{Pitfalls}

Complications commonly seen after TMA are wound dehiscence, surgical site infection, revision amputations, and ankle equinus. Anthony et al. ${ }^{8}$ reported that $82 \%$ of TMA patients required second surgery due to complications and Pollard et al. ${ }^{9}$ interpreted $32 \%$ of TMAs required revision amputations.

Patient selection and preoperative workup are the two most important steps in prevention of these complications. According to the literature, most commonly diabetics required TMA due to ischemia, infection, or both. Early admission in hospital for random blood sugar (RBS) control, antibiotic administration, and/or surgical

\begin{abstract}
${ }^{1-3}$ Department of Orthopaedics, Postgraduate Institute of Medical Education and Research, Chandigarh, India

Corresponding Author: Aman Hooda, Department of Orthopaedics, Postgraduate Institute of Medical Education and Research, Chandigarh, India, Phone: +919646003620, e-mail: amanhooda_10@yahoo.com

How to cite this article: Kumar D, Hooda A, Thami T. Evolution of Principles of Transmetatarsal Amputations: How We Do It Today. J Foot Ankle Surg (Asia Pacific) 2020;7(2):73-76.

Source of support: Nil

Conflict of interest: None
\end{abstract}

therapy for wound abscess showed great results in preventing revision amputations. ${ }^{10}$ Limb vascularity should be assessed preoperatively with computed tomography (CT) angiography, transcutaneous pulse oximetry, ankle-brachial index (ABI), and systolic skin pressure (SPP); a revascularization or bypass procedure should be recommended if vascular channels are found amenable to repair. ${ }^{11} \mathrm{~A}$ retrospective analysis by Pinzur et al. ${ }^{12}$ revealed a $92 \%$ success rate after TMA and patients with minimum $\mathrm{ABI}$ of $0.45-0.50$, total lymphocyte count more than $1.5 \times 10^{3} \mu \mathrm{L}$, and serum albumin more than $3 \mathrm{~g} / \mathrm{dL}$ had relatively better outcome postoperatively. Historically, the problem of wound dehiscence is quite frequent after TMA but its incidence can be kept to a minimum with proper planning of skin incision for preservation of maximal length of a plantar flap, for optimal coverage of metatarsal heads and to avoid peak pressure points. ${ }^{1}$ In cases with a higher risk of infections or contamination, open guillotine TMA stump ${ }^{5}$ or use of negative pressure wound therapy (NPWT) ${ }^{13}$ showed promising results in the literature. Another significant problem associated with TMA is ankle equinus due to shortened lever arm of foot. Muscular imbalance occurs due to unopposed gastrocnemius action with the loss of extensor hallucis longus and extensor digitorum longus. Additional procedures like split tibialis anterior transfer, tendo-Achilles lengthening, or gastrocnemius recession have been successfully combined with TMA to prevent ankle equinus. ${ }^{14,15}$

\section{Postoperative Care}

In the 1950s, patients were advised to wear standard shoes following TMA with a shoe filler in the form of lamb's wool. ${ }^{16,17}$ 
In the 1960s, Bauman et al. ${ }^{18}$ stated that "a foot without toes is urgently in need of protection". He did a comparative study of shoe modification in patients following TMA and proved that a rocker bottom sole with the apex at the center of the shoe was the most effective in reducing plantar forefoot pressures during the late stance and push-off phases of gait. Despite these results, the old method of shoe filler in a standard shoe persisted in view of the fact that foot following TMA was not different from the normal due to the preservation of a larger length of the foot. ${ }^{19}$ In another study, Mueller et al. ${ }^{20}$ in 1997 compared various orthotics, shoe gear, and braces in patients following TMA and they found that the custom full-length shoe with a rocker bottom sole and total contact insert was most effective in terms of cosmesis, functional capacity, and patient adherence. The proper combination of these will augment stability, aid in ambulation, and protect the residual forefoot from increased pressure and shear strain.

\section{How We Do It}

\section{Preoperative Management}

Appropriate patient selection, with strict indications, is still the most important factor for the success of TMA. The most common indication is a diabetic foot with infection limited to the distal part of foot followed by unsalvageable mangled forefoot, local gangrene of multiple digits, and in some cases with nonhealing ulcers over the interweb areas of toes. The patients are adequately informed about the procedure and the possible complications. Since these patients are generally diabetic, they should be admitted to the hospital 1 week prior to the surgery for preoperative assessment and glycemic control. Investigations should include RBS and $\mathrm{Hb} 1 \mathrm{Ac}$ measurement. Oral hypoglycemic drugs and intravenous insulin by the sliding scale can be administered during the perioperative period to control blood sugar values. The extent of infection should be ascertained clinically and necessary investigations like total WBC count, C-reactive protein, serum albumin, total lymphocyte count, and cultures should be ordered. If necessary, local wound debridement and systemic antibiotics should be given. Assessment of vascular status with Doppler ultrasound, transcutaneous pulse oximetry, ABI, and SSP should be performed. CT angiography will be performed in limited cases when there is a plan of revascularization or bypass by a vascular surgery team. Peripheral sensory neuropathy is routinely assessed with 5.07/10 g monofilament, whereas nerve conduction studies are kept limited to special cases. The operative leg should not be shaved in the preoperative period and instead washed with chlorhexidine solution and draped 12 hours prior to the surgery.

\section{Intraoperative Care}

The surgery is usually performed under regional or general anesthesia and tourniquet use should be avoided in diabetic foot and in cases of vascular insufficiency. The leg is prepared aseptically and draped. After marking the incision, a curved incision is made over the dorsum of the foot extending from the middle of the base of first metatarsal to the middle of the base of fifth metatarsal in a single stroke (Fig. 1A). The incision is centered the level of the osteotomy. The incision over the plantar side should be at the level of metatarsophalangeal (MTP) joint so that adequate skin would be available for coverage and tension-free closure of the stump, whenever there is a deficient dorsal flap (Fig. 1B). The proposed osteotomy should be at the level of proximal part of metatarsal at an angle of $45^{\circ}$ in the coronal plane to avoid pressure points and should progress from the fifth metatarsal to first metatarsal (lateral to medial) with formation of a smooth curve. Each successive cut is $2-3 \mathrm{~mm}$ longer than the lateral metatarsal with the help of an oscillating saw. The distal parts of the metatarsal should be lifted dorsally, and soft tissue should be cleared with electrocautery or surgical knife to complete the amputation by keeping the dissection in close proximity to the bone. The flexor tendons and nerve should be cut with a sharp knife after pulling them out for $1 \mathrm{~cm}$ to prevent formation of a neuroma. Muscles should be beveled in the plantar direction followed by smoothening of edges of the bone. In the end, after achieving the hemostasis, check for the length and viability of plantar flap. The wound is closed in a tension-free manner using the moccasin technique with non-absorbable sutures (Fig. 1C). In cases of severe contamination or high-risk cases, a guillotine TMA stump is left, which is usually combined with NPWT. In our institute, a gastrocnemius recession is usually performed after TMA to prevent ankle equinus.

\section{Postoperative Management}

A below-knee slab should be given in the postoperative period for better wound healing. Antibiotics are usually started in the perioperative period and continued up to 5 days postoperatively and the duration can be individualized as per the need. In cases of guillotine or NPWT, wound closure with or without split skin graft can be performed on a postoperative day 5. Alternate day sterile dressing is to be performed till the wound heals. Sutures can be removed safely around 3 weeks postoperatively in most cases but may be delayed until satisfactory wound healing. Protected weight-bearing is usually advised in a slab or custom shoe for 3-4 weeks until the edema resolves, to prevent infection and wound dehiscence. After 4 weeks, ambulation in sport's shoes with or without filler is advised.

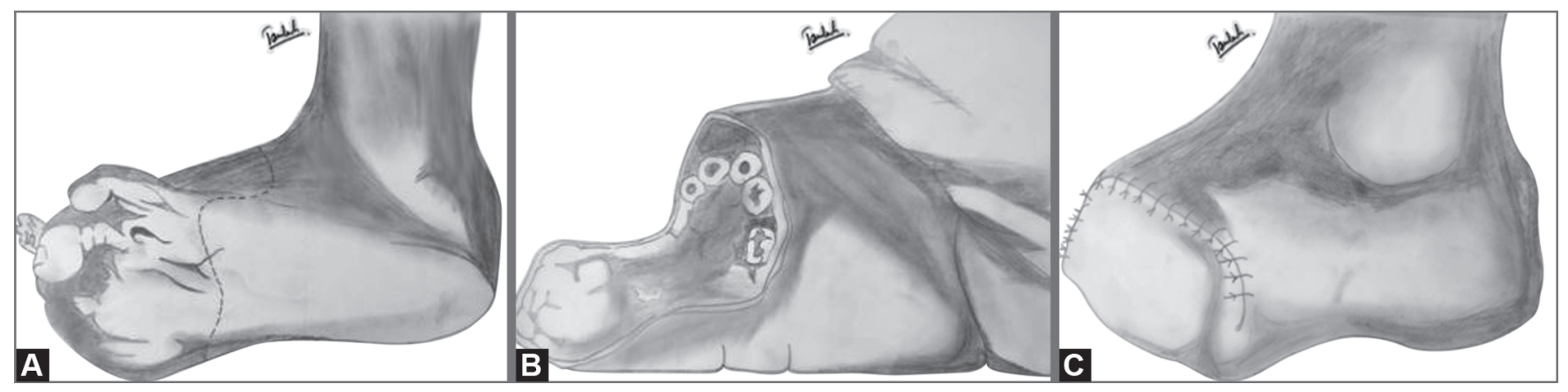

Figs 1 A to C: (A) Line diagrams describing the marking of the incision; (B) Adequate exposure with long plantar flap; (C) Tension-free closure with nonabsorbable sutures 
Flowchart 1: Treatment algorithm of transmetatarsal amputation

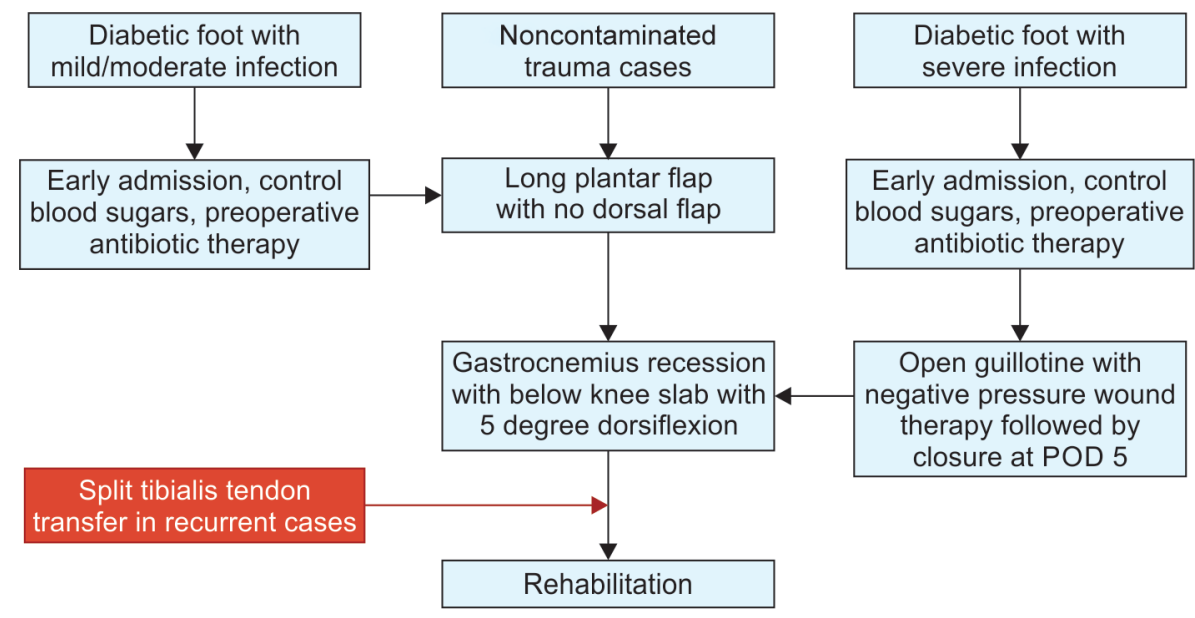

\section{What has Changed?}

In recent years, the principles of TMA have been changed to minimize the complications. Initially, the approach was to keep a long plantar flap with no dorsal flap by McKittrick et al. ${ }^{3}$ which was later modified by Durham et al. ${ }^{6}$ as they kept the TMA open or guillotine in treatment of severe foot infections with a larger plantar flap which was closed later after a course of antibiotics. In 2011, Terashi et al. ${ }^{7}$ described a modification in which he preserved the soft tissues between the metatarsal bones and applied it to the distal stumps. They successfully closed 11 wounds with this new technique.

Wound management has also evolved in recent years and decreased the incidence of revision amputations. Complications like wound dehiscence and revision amputation were common earlier, Armstrong et al. ${ }^{13}$ published excellent outcomes in 2005 with the use of NPWT in complicated diabetic foot amputations.

In the past, the ankle equinus following TMA was neglected, ultimately resulting in functional limitation. The principles of prevention of equinus have evolved from the application of belowknee slab with the ankle in slight dorsiflexion to surgical procedures like gastrocnemius recession, tendo-Achilles lengthening, and tibialis anterior transfer. ${ }^{13,14}$ The combined method of gastrocnemius recession followed by below-knee slab with the ankle in $5^{\circ}$ dorsiflexion showed excellent results in our practice and procedures like split tibialis anterior transfer were limited to recurrent cases.

In postoperative care, earlier a standard shoe with a filler was thought to be adequate in the 1950s. ${ }^{16,17}$ But the work of Mueller et al. ${ }^{20}$ proved that use of a total contact insert in a custom-molded high type shoe with a rocker bottom sole was more effective in decreasing pressure to both the forefoot and the hindfoot resulted in better cosmesis. Current and future surgeons should be encouraged to work with orthotics and patients should be educated thoroughly regarding do's and don'ts to minimize complications in the future. In the end, we summarize the treatment algorithm for TMA (Flowchart 1).

\section{References}

1. McCallum R, Tagoe M. Transmetatarsal amputation: a case series and review of the literature. J Aging Res 2012;2012:797218. DOI: $10.1155 / 2012 / 797218$.
2. Bernard C, Huete C, Schwindt CD, et al. Transmetatarsal amputation. Ortho Clin North Am 1973;4:31-42.

3. McKittrick LS, McKittrick JB, Risley TS. Transmetatarsal amputation for infection or gangrene in patients with diabetes mellitus. Ann Surg 1949;130(4):826-842. DOI: 10.1097/00000658-19491000000019.

4. Mueller MJ, Sinacore DR. Rehabilitation factors following transmetatarsal amputation. Phys Ther 1994;74(11):1027-1033. DOI: 10.1093/ptj/74.11.1027.

5. Sanders LJ. Transmetatarsal and midfoot amputations. Clin Podiatr Med Surg 1997;14(4):741-762.

6. Durham JR, McCoy DM, Sawchuk AP, et al. Open transmetatarsal amputation in the treatment of severe foot infections. Am J Surg 1989;158(2):127-130. DOI: 10.1016/0002-9610(89)90360-7.

7. Terashi H, Kitano I, Tsuji $\mathrm{Y}$, et al. A modified transmetatarsal amputation. J Foot Ankle Surg 2011;50(4):441-444. DOI: 10.1053/ j.jfas.2011.03.018.

8. Anthony T, Roberts J, Modrall JG, et al. Transmetatarsal amputation: assessment of current selection criteria. Am J Surg 2006;192(5):e8-e11. DOI: 10.1016/j.amjsurg.2006.08.011.

9. Pollard J, Hamilton GA, Rush SM, et al. Mortality and morbidity after transmetatarsal amputation: retrospective review of 101 cases. J Foot Ankle Surg 2006;45(2):91-97. DOI: 10.1053/j.jfas.2005.12.011.

10. Landry GJ, Silverman DA, Liem TK, et al. Predictors of healing and functional outcome following transmetatarsal amputations. Arch Surg 2011;146(9):1005-1009. DOI: 10.1001/archsurg.2011.206.

11. Miller N, Dardik $\mathrm{H}$, Wolodinger F, et al. Transmetatarsal amputation: the role of adjunctive revascularization. J Vasc Surg 1991;13(5): 705-711. DOI: 10.1016/0741-5214(91)90357-Z.

12. Pinzur M, Kaminsky M, Sage R, et al. Amputations at the middle level of the foot. A retrospective and prospective review. J Bone Joint Surg Am 1986;68(7):1061-1064. DOI: 10.2106/00004623-198668070-00013.

13. Armstrong DG, Lavery LA. Negative pressure wound therapy after partial diabetic foot amputation: a multicentre, randomised controlled trial. Lancet 2005;366(9498):1704-1710. DOI: 10.1016/ S0140-6736(05)67695-7.

14. Armstrong DG, Stacpoole-Shea $S$, Nguyen $H$, et al. Lengthening of the achilles tendon in diabetic patients who are at high risk for ulceration of the foot. J Bone Joint Surg Am 1999;81(4):535-538. DOI: 10.2106/00004623-199904000-00011.

15. Sharrard WJW, Bernstein S. Equinus deformity in cerebral palsy. A comparison between elongation of the tendo calcaneus and gastrocnemius recession. J Bone Joint Surg Br 1972;54(2):272-276. DOI: 10.1302/0301-620X.54B2.272.

16. Warren R, Crawford ED, Hardy IB, et al. The transmetatarsal amputation in arterial deficiency of the lower extremity. Surgery 1952;31(1):132-140. 
17. Pedersen $\mathrm{HE}$, Day AJ. The transmetatarsal amputation in peripheral vascular disease. J Bone Joint Surg Am 1954;36-A(6):1190-1198. DOI: 10.2106/00004623-195436060-00008.

18. Bauman JH, Girling JP, Brand PW. Plantar pressures and trophic ulceration. An evaluation of footwear. J Bone Joint Surg 1963;45(4):652-673. DOI: 10.1302/0301-620X.45B4. 652.
19. Garbalosa JC, Cavanagh PR, Wu G, et al. Foot function in diabetic patients after partial amputation. Foot Ankle Int 1996;17(1):43-48. DOI: $10.1177 / 107110079601700110$.

20. Mueller M, Strube M, Allen B. Effect of six types of footwear on peak plantar pressures in patients with diabetes and transmetatarsal amputation. Clin Biomech 1997;12(3):S3. DOI: 10.1016/S02680033(97)88308-8. 